\title{
Mechanical properties of Gelatin -Hydroxyapatite composite for bone tissue engineering
}

\author{
M. J. Hossan ${ }^{1}$, M. A. Gafur ${ }^{2}$, M. M. Karim ${ }^{1}$ and A. A. Rana ${ }^{1 *}$ \\ ${ }^{I}$ Department of Applied Chemistry and Chemical Engineering, University of Dhaka, Dhaka-1000, Bangladesh \\ ${ }^{2}$ PP and PDC, Bangladesh Council of Scientific and Industrial Research (BCSIR), Dhaka, Bangladesh.
}

\begin{abstract}
In this study, hydroxyapatite (HAp) and gelatin (GEL) scaffolds were prepared to mimic the mineral and organic component of natural bone. The raw material was first compounded and resulting composite were molded into the petridishes. Using Solvent casting process, it is possible to produce scaffolds with mechanical and structural properties close to natural trabecular bone.The mechanical properties of composites were investigated by Thermo-mechanical analyzer (TMA), Vickers microhardness tester, Universal testing machine. It was observed that the composite has maximum tensile strength of $37.13 \mathrm{MPa}$ ( oven drying) and \% elongation of 7.68 (Oven drying) and 2.04 (Natural drying) at 15\% of Hap respectively. These results demonstrate that the prepared composite scaffold is a potential candidate for bone tissue engineering.
\end{abstract}

Keywords: Hydroxiapatite; Thermomechanical analysis; Tensile strength; Tensile elongation

\section{Introduction}

Biomaterials play a very important role in tissue engineering, which is aimed to provide replacements for tissues and organs which have been damaged or lost as a consequence of disease, aging or accident (Safinya et al., 1996). A part of biomaterials is designed for replacing bone and/or inducing bone formation. From the biological perspective the natural bone matrix is a combination of organic/inorganic composite materials and consists of a naturally occurring polymer (collagen) and a biological mineral (apatite) (Chu and Liu, 2008). Further blending with inorganic materials can modify the mechanical properties as well as the degradation rates of the materials. The designed composite scaffold should combine the advantages of both components. Moreover, the natural composite material should have an excellent balance between strength and toughness, both of which should be superior to those of the individual components (Ma, 2008). Therefore, instead of using a single material type for synthesis it is a natural strategy to combine polymers and inorganic ceramic i.e. hydroxyapatite (HAp) to fabricate scaffolds that meet all the requirements desired for particular applications in tissue engineering. Thus polymer/inorganic composite scaffolds have attracted the attention of researchers.

Over the past decades, growing interest has been focused on the development of inorganic-organic hybrid biomaterials in order to mimic natural materials' composition and structure. A minority component of organic macromolecules in ceramics such as proteins or polymers are usually used to control nucleation and growth of inorganic crystals, and thus to improve microstructure and physical properties. This related methodology has a great potential for the design and engineering of novel biomaterials with special functionalities (Safinya et al. 1996).

According to a literature review the compressive strengths of cortical and cancellous bone are in the ranges 100-230 and 2-12 MPa, whereas the Young's moduli are in the ranges 7-30 and 0.5-0.005 GPa, respectively (Hutmacher et al., 2007). Among the commonly used polymers, poly (lactic acid) degrades within the human body to form lactic acid, a naturally occurring substance which is easily removed from the body material. One of the main disadvantages of biodegradable polymers used in tissue engineering is their poor mechanical properties. Thus, there have been many studies to improve the mechanical properties of biodegradable polymers by incorporation of other materials (Gontard et al., 1993). In biomineralized tissues as bone, teeth and calcified tendons, the collagen matrix is stiffened by the apatitic crystals which act as filler particles (Vincent et al., 1990). Due to the close structural relationship between the two components, the resulting composite material exhibits

*Corresponding author: E-mail: rana_3786@yahoo.com 
mechanical properties which depend on the degree of calcification and on the distribution and orientation of both collagen fibrils and inorganic crystals (Evans et al., 1973; Ascenzi et al., 1994). Thus, in order to obtain a better combination of biocompatibility, biodegradation and superior mechanical strength, composites of polymer and bioactive ceramics have been considered for bone tissue engineering.

Calcium phosphates, mainly in the form of hydroxyapatite, have been widely used in bone implants. As bone defect fillers, these ceramics are utilized in powder and block forms. These materials exhibit several problems of handling and fabrication, which can be overcome by mixing with a suitable binder. Recently, a great interest has been focussed on the use of composites of degradable biopolymers, such as fibrin, collagen and gelatin, with inorganic powders, as bone fillers (Yao et al., 1996). Several authors have investigated equivalent materials and the most recent attempts have used polymer additives such as poly(lactic acid) (PLA), poly(acrylic acid) (PAAc), collagen, and gelatin due to the efficiency of their contained calcium binding properties (Chang et al., 2003; Schiller et al., 2003; Chian Liou et al., 2003; Spanos et al., 2002). As biomaterial, gelatin displays several attractive: it has not shown antigenity, it is completely resorbable in vivo, its physicochemical properties can be suitably modulated (Yao et al., 1996; Fakirov et al., 1996). Furthermore, its adhesiveness and plasticity properties make gelatin a suitable matrix for ceramic composites.

The gelatin-HAp composite was prepared through casting method as it seems to be a rather simple and economical approach in the preparation of porous scaffold materials (White et al., 1972). We have discussed the synthesis and characterization of the mentioned composite in our previous paper (Hossan et al., 2014). Upon finding promising results, herein we like to discuss the mechanical properties such as Tensile strength analysis, \% Elongation break, Vickers micro-hardness test analysis, thermo-mechanical analysis of this composite. The aim of this paper is to evaluate the mechanical and thermal properties of the composite for application in bone tissue engineering.

\section{Materials and methods}

\section{Materials}

Reagent grade $\mathrm{Ca}(\mathrm{OH})_{2}$ (96\% pure) and ortho-phosphoric acid (85\%) were purchased from Loba Chemie (india) and Merck (Germany) respectively. Gelatin was purchased from Oxoid Ltd., Basingstoke (England). All the chemicals were used as received and without further purification. All the solutions were prepared using double distilled water.

\section{Method}

\section{Preparation of HAp powder}

Preparation of gelatin-hydroxyapatite composite scaffold was started by preparing hydroxyapatite crystallites by a chemical precipitation method[ (Kumar and Prakash , 2004). $1 \mathrm{~mol} \mathrm{Ca}(\mathrm{OH})_{2}(96 \%$ pure) was added to $100 \mathrm{~mL}$ of distilled water in a beaker and stirred at reaction temperature for $1 \mathrm{~h}$. On the other hand $100 \mathrm{~mL}$ solution of $0.6 \mathrm{~mol} \mathrm{H}_{3} \mathrm{PO}_{4}$ was taken in another beaker. Appropriate amounts $(\mathrm{Ca} / \mathrm{P}$ ratio of 1.67) of orthophosphoric acid solution was added gradually ( 2 drops per second) from burette in the beaker with stirring at room temperature. , The appearance of milky white coloration is an indication for the formation of hydroxyapatite (Sinha et al. 2003). After reaching this point, the suspension was stirred further for $2 \mathrm{~h}$, before it was left to settle overight (about $15 \mathrm{~h}$ ) at room temperature. The HAp precipitates were then separated by filtration. At some times the wet cakes were washed with hot distilled water, dried in a oven at $100^{\circ} \mathrm{C}$ and ground to fine powder.

\section{Preparation of Gelatin - HAp composite}

The slurry composite was prepared using solvent casting method. As dry GEL is essentially intractable material, it can readily become castable when transformed into a sol-gel state by dissolving in water up to about $5-30 \mathrm{wt} \%$. In order to have a homogenous and strong composite, the HAp particles finer than $150 \mu \mathrm{m}$ were obtained using Ultrasonicator. Definite GEL content of $12.33 \mathrm{wt} \%$ was dissolved in DI water at $45^{\circ} \mathrm{C}$. Then the $5 \mathrm{wt} \%, 10 \mathrm{wt} \%, 15 \mathrm{wt} \%$ and $20 \mathrm{wt} \%$ HAp contents were added to prepare four different composites. The reinforced slurry composite was then heat treated under constant mixing at $40^{\circ} \mathrm{C}$ for $1 \mathrm{~h}$. The slurry was deagglomerated by magnetic stirring. The temperature was monitored continuously. The slurry was immediately transferred into definite sized petridishes. The molds were frozen at $-40^{\circ} \mathrm{C}$ and then freeze dried for $3 \mathrm{~h}$ for solvent (DI) removal., The white composites were then removed and placed in room temperature for $24 \mathrm{~h}$. For statistical analysis in all assays, five samples of each type were investigated and the average was reported.

\section{Thermo mechanical analyzer}

Thermomechanical analysis have been done by TMA/SS 6300,SII Nano Technology, Japan, system controlled by an EXSTAR 6300 controller.TMA studies have been carried out on freeze dried Gelatin-Hap composite sample in different weight. Experiments have been performed using simultaneous TMA analysis by heating the sample at $5^{\circ} \mathrm{C} / \mathrm{min}$ in the temperature range of $25^{\circ} \mathrm{C}$ to $100^{\circ} \mathrm{C}$ in nitrogen atmosphere. 


\section{Mechanical Test}

Tensile properties such as tensile strength (TS) and per cent elongation at break $(\mathrm{Eb})$ of the cured films were measured with Universal Testing Machine (Hounsfield Series S, UK) with a cross head speed of $10 \mathrm{~mm} / \mathrm{min}$. The load range of $500 \mathrm{~N}$ and the gauze length $20 \mathrm{~mm}$ were used throughout the experiment. Microhardness of the compressed specimen was measured by means of a Vickers microhardness tester (HMV-2, Shimadzu Corporation, Japan). A pyramidal diamond indenter was applied to the specimen surface under different load of 0.098 $\mathrm{N}, 0.2452 \mathrm{~N}$ and 0.4903 for $15 \mathrm{~s}$ respectively. Diagonal length of the indentation was measured through a micometric eyepiece with an objective lens of $40 x_{-}$. The tests were repeated five times for each sample.

\section{Results and discussion}

\section{Thermomechanical analysis(TMA)}

TMA was used to observe the thermal expansion of material and composite, softening point of materials, bending properties of materials and modulus of elasticity. Amount of probe movement vs temperature for softening point analysis was shown in figures 1-3. An almost linear trend in increasing softening point with increasing amount of HAp blended with gelatin has been observed. It was observed that highest softening point of $47.16^{\circ} \mathrm{C}$ was achieved with $20 \%$ HAp blended gelatin. However there was no significant increase in softening point above $10 \%$ HAp blended gelatin composite. The change in molecular interaction and different thermal response of the hydroxyapatite and gelatin might have shifted the softening point of film. Thus thermal stability of composite improved with increasing amount of Hap with gelatin.

\section{Tensile strength analysis of Gelatin-HAP composite}

Tensile strength (TS) is very important property in selecting diverse application of composite materials. The results of TS values of the natural drying and oven drying gelatin films blended with HAp are plotted in Figure 4.

It has been observed that the tensile strength increased linearly with increasing \% of HAp at oven drying and natural drying respectively due to cross-linking between GelatinHAp. However, the naturally dried composite films showed significant higher tensile strength than that of the oven dried films. For instance, naturally dried film with 5\% HAp showed about $40 \%$ higher tensile strength than the oven dried film. This may be explained by the fact that, deformation of the helix structure of gelatin occurred above $60^{\circ} \mathrm{C}$;

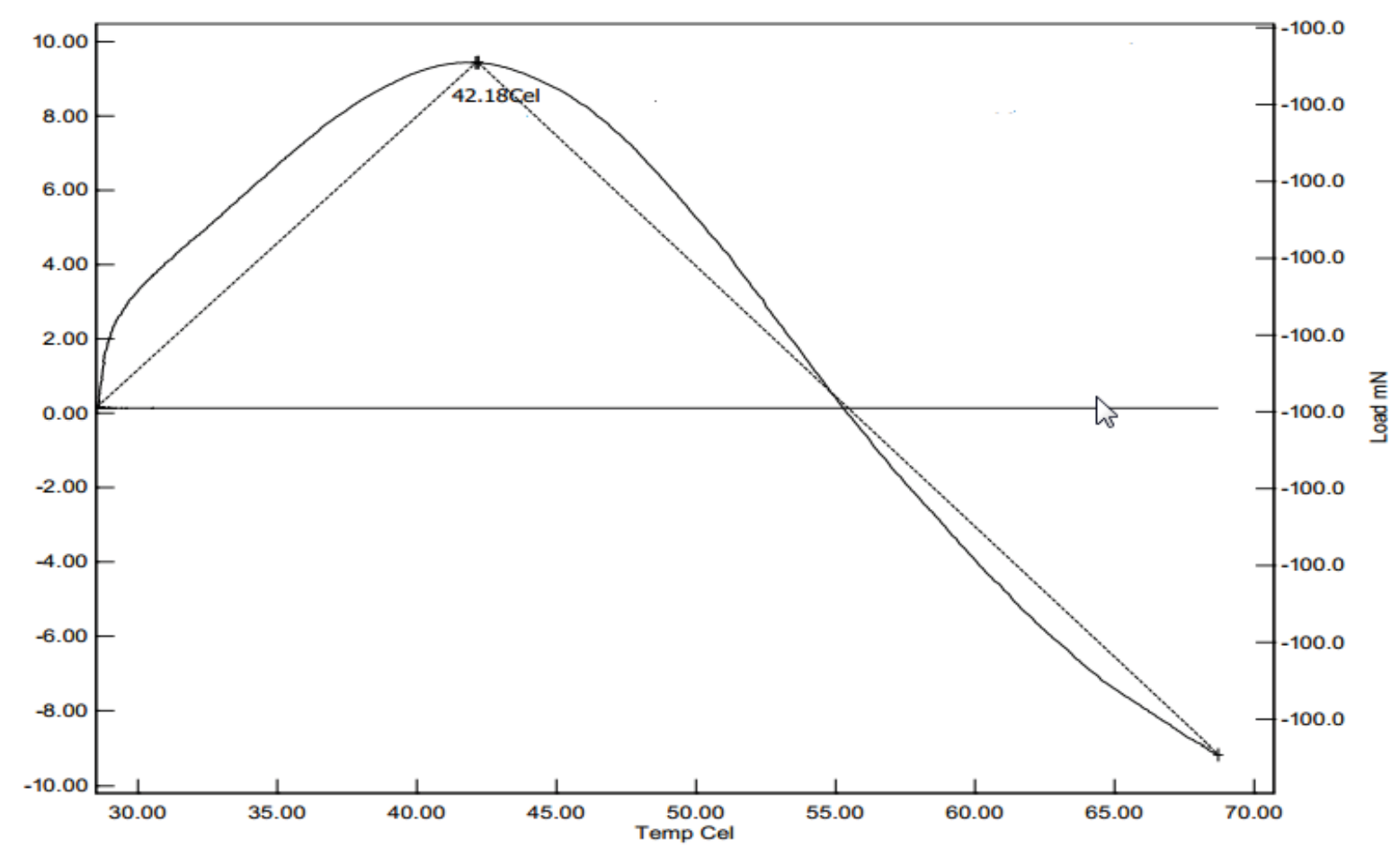

Fig. 1. Thermomechanical thermogram of Pure Gelatin 


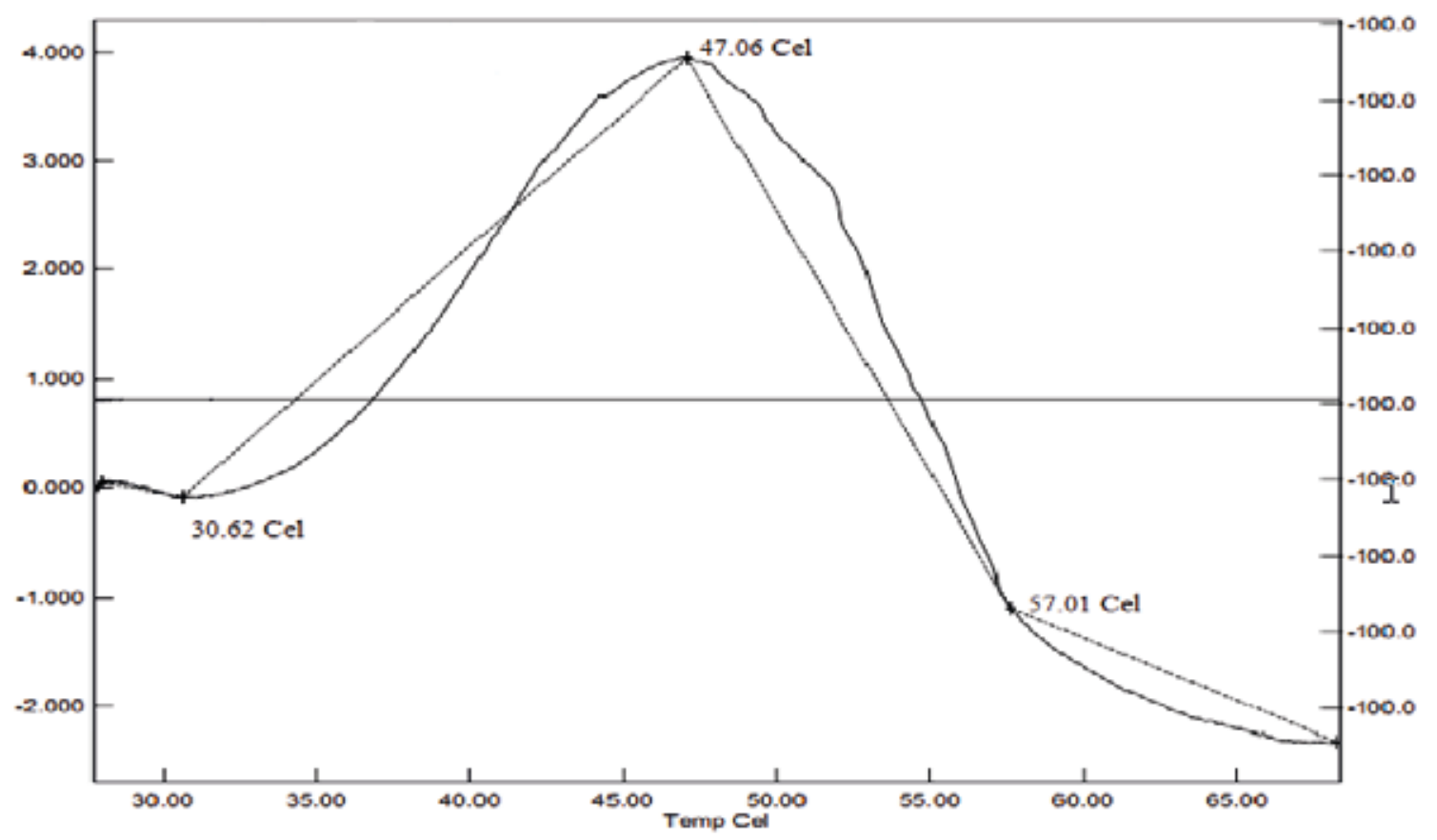

Fig. 2. Thermomechanical thermogram of Gelatin-HAp composite containing $10 \mathrm{wt} \%$ HAp

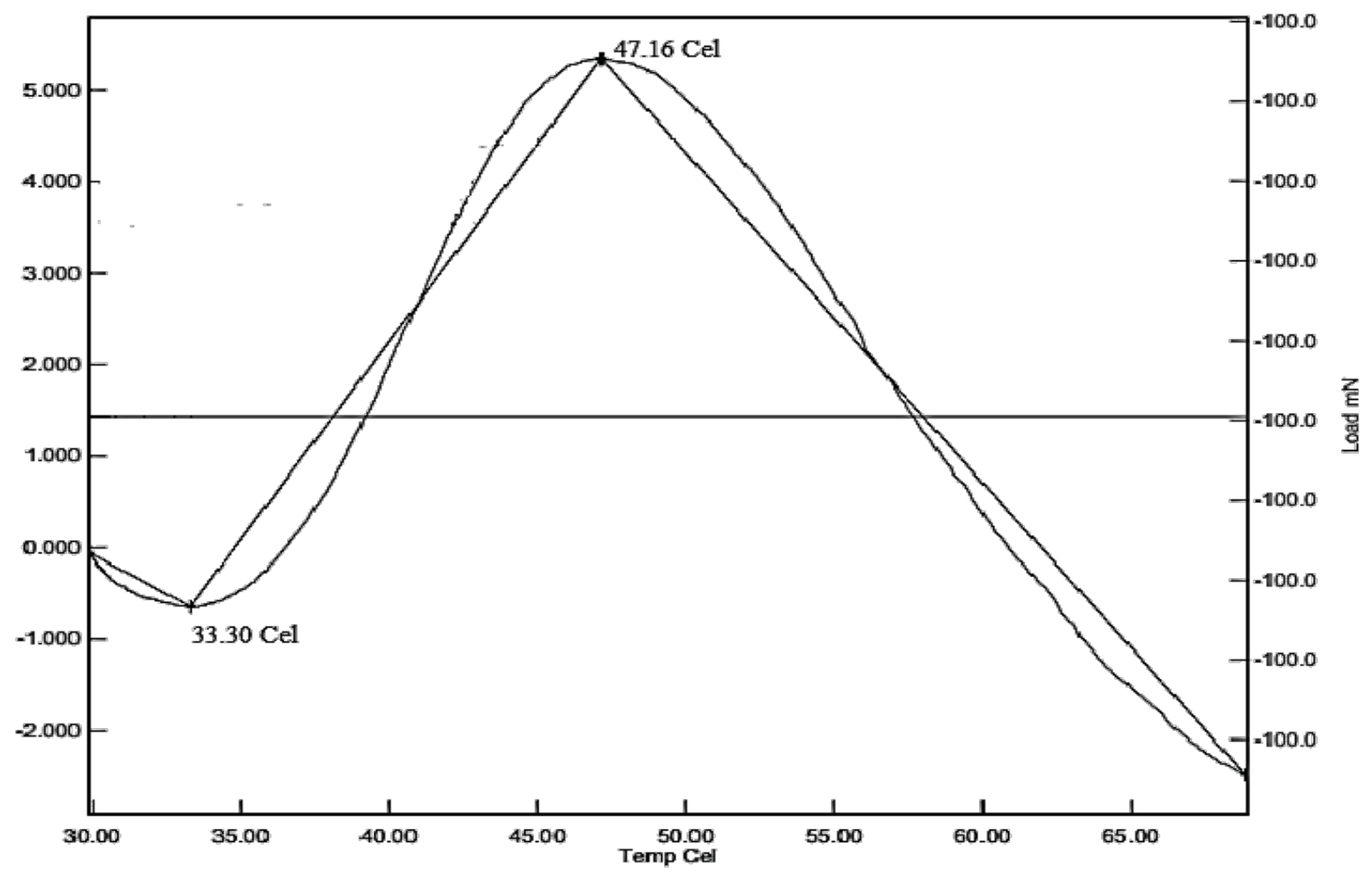

Fig. 3. Thermomechanical thermogram of Gelatin-HAp composite containing $20 \mathrm{wt} \%$ HAp. 


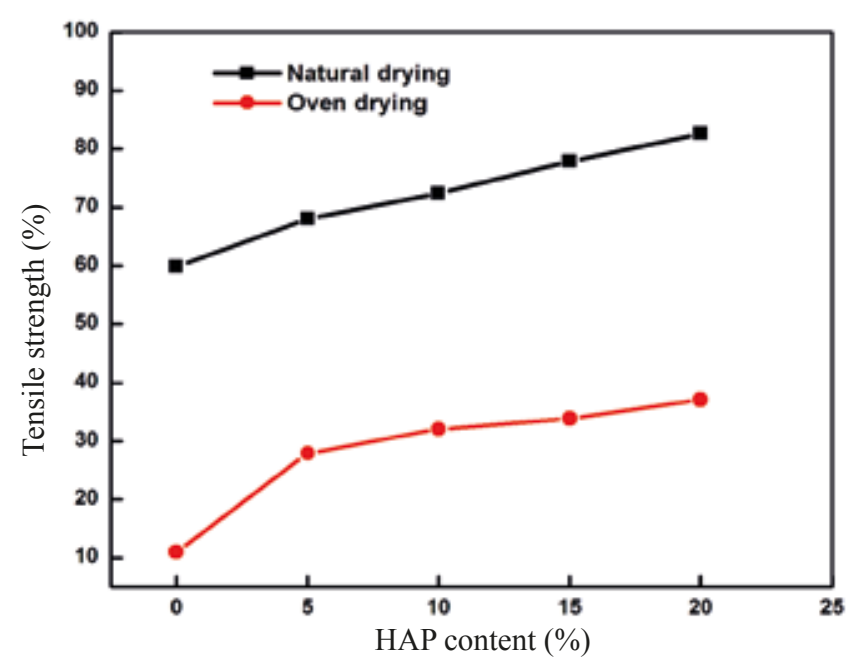

Fig. 4. Tensile strength of HAp- Gelatin composite containing different \% of HAp.

consequently decrease the strength of gelatin itself with the quick removal of water. On the other hand, incorporation of ceramic HAp in highly oriented (naturally dried) gelatin structure significantly increases the tensile strength.

\section{$\%$ Elongation of Gelatin- HAp composite}

The results of elongation at break (\%) of the naturally dried and oven dried films against HAp composition were plotted in Figure 5. it was observed that percent elongation at break decreases for oven dried samples sharply due to incorporating HAp. It is also observed from the figure that for oven dried film, the lowest elongation at break was found to be $6.43 \%$ for $20 \%$ HAp containing gelatin film which is $10.3 \%$ lower

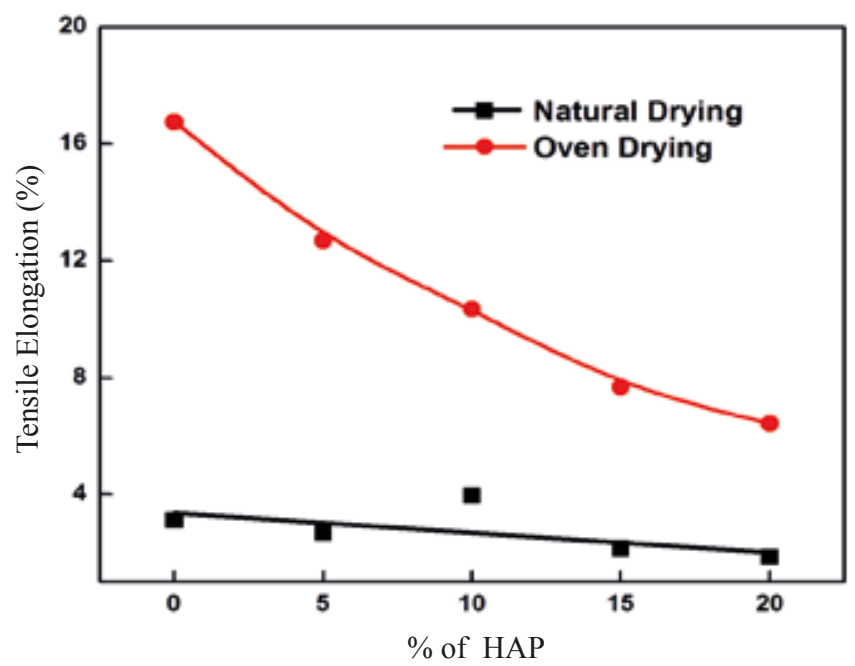

Fig. 5. Tensile elongation of HAp-Gelatin composite containing different \% of HAp. than pure gelatin film. On the other hand, naturally dried film showed very slow decrease in tensile elongation with the incorporation of HAp. These results were very supportive with that of tensile strength results. As mentioned earlier, at higher temperature, the natural helix structure of gelatin is destroyed which eventually increase the plasticizing property of the gelatin. This increased plastisizing effect made the gelatin comparatively softer. Thus incorporation of inorganic HAp sharply decrease the tensile elongation and increase the tensile strength respectively.

\section{Vickers Microhardness test Analysis}

Microhardness testing of metals, ceramics, and composites is useful for a variety of applications for which 'macro' hardness measurements are unsuitable The diagonal length of the micro Vickers indentations, is much larger than the average aggregate size, i.e. 8.0-9.5 $\mu \mathrm{m}$. On this basis, we may reasonably assume that the microhardness reflects the strength of the connections among aggregates, rather than within an aggregate. Variation in the Vickers microhardness of the compressed specimen versus Hydroxyapatite content under different working load was shown in figure 6. It was evident from the figure that the Vickers microhardness

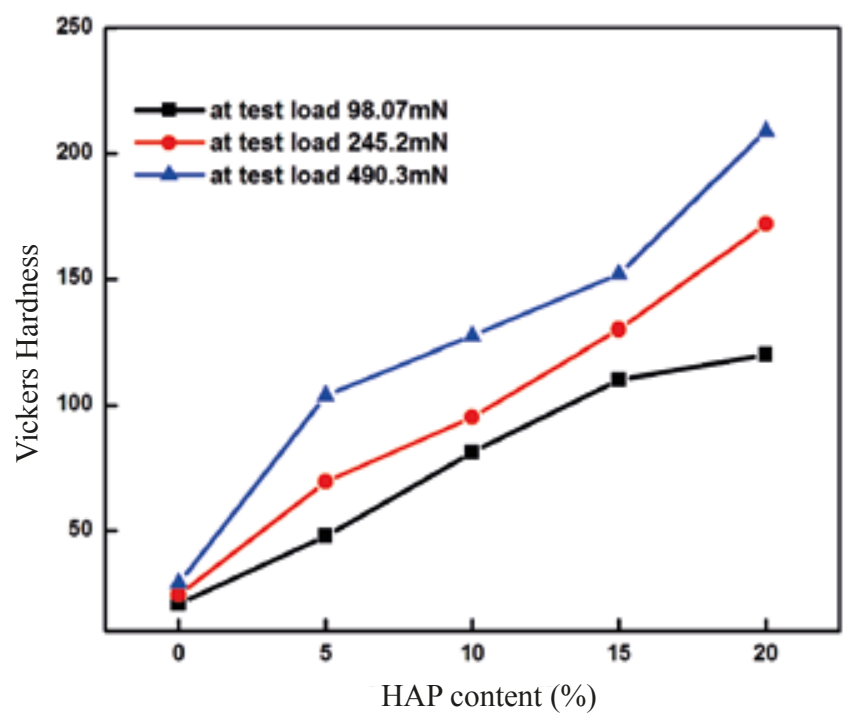

Fig. 6. Vickers microhardness of the compressed specimen versus Hydroxyapatite content under different working load

increases about 6-7 times with the increase of HAp content of $20 \%$. It is commonly accepted that cross linking of the ceramic material with gelatin increases with increasing percentage of HAp and consequently increases the hardness of the composite material. 


\section{Conclusion}

Thermal and mechanical properties of gelatin-HAp composite significantly depend on the ratio of gelatin to Hydroxyapatite composition. TMA data revealed that high thermal stability with higher softening point were achieved with the increase in HAp content in the composite film. Tensile strength as well as micro hardness of the composite increased with the increase of HAp. However naturally dried composite in all composition has more tensile strength and hardness compare to oven dried one as more cross-linking occur in the naturally dried one. on the otherhand, elongation percentage of both natural and oven dried composite decreased with the increase of HAp content. Tensile elongation of the oven dried film significantly decreased as compared with naturally dried one due to structural deformation and plasticizing effect of gelatin. From these results, it could be suggested that gelatin and hydroxyapatite films of different ratios appears to have potential as bone tissue engineering because of their biodegradability and biocompatibility. However more thorough investigations were required for accurate evaluation of the mechanical properties of this composite.

\section{Acknowledgement}

The authors acknowledge the technical support provided by Pilot Plant and Process Development Center of BSCSIR, Bangladesh.

\section{References}

Ascenzi A, Baschieri P, Benvenuti A.(1994), The torsional properties of single selected osteons, J Biomechanics, 27: $875-84$.

Chang M.C, Ko C.C, Douglas W. H.(2003), Biomater. 24(17): 2853-2862

Chian Liou S. Z., Chen S. Y., Liu. D. M.,(2003), Biomater., 24(22): 3981-3988

Chu PK and Liu X (2008). Biomaterials fabrication and processing handbook. (Boca Raton, FL: CRC Press) 2008.

Evans FG (1973), Mechanical Properties of Bone. (Springfield, Ill:Charles C. Thomas Publisher), 1973.

Fakirov S, Sarac Z, Anbar T, Boz B, Bahar I, Evstatiev, M, Apostolov AA, Mark JE, Kloczkowski A.(1996), Mechanical properties and transition temperatures of cross-linked oriented gelatin, Colloid Polym Sci., 274: 334-41
Gontard, N., Guilbert, S., \& Cuq, J. L. (1993), Water and glycerol as plasticizers affect mechanical and water vapor barrier properties of an edible wheat film, Journal of Food Science, 58: 206-211

Hossan M.J, Gafur M. A, Kadir M. R and Karim M.M (2014), Ijet-Ijens Vol: 14 No: 01

Hutmacher DW, Schantz JT, Lam CXF, Tan KC, Lim TC (2007), State of the art and future directions of scaffold-based bone engineering from a biomaterials perspective, J Tissue Eng Regen Med, 1: 245-260

Kumar R. and Prakash K. H. (2004), Langmuir, 20: 5196-5200.

Ma PX (2008). Biomimetic materials for tissue engineering. Adv Drug Deliv Rev , 60: 184-98.

Safinya CR and Addadi L.( 1996). Current Opinion in Solid State and Materials Science; Biomaterials. 1: 387-91

Schiller C., Matthias E.,(2003), Biomater. 24(12): 2037-2043

Sinha A., Nayer S., Agrawak A. C.(2003), J. Am. Ceram. Soc., 86: 357-359

Spanos N., Deimede V., Koutsoukos P. G.(2002), Biomater., 23(3): 947-953.

Vincent J (1990), Structural Biomaterials. (Princeton: Princeton University Press) 1990

White R.A., Weber J.N., White E.W., (1972), Replamineform:A new process for preparing porous ceramics, metal and polymer prosthetic materials, Science, 176: 922-24.

Yao CH, Sun JS, Lin FH, Liao CJ, Huang CW.(1996), Biological effects and cytotoxicity of tricalcium phosphate and formaldehyde crosslinked gelatin composite. Mater Chem Phys, 45: 6-14

Received: 03 November 2014; Revised: 04 March 2015 Accepted: 24 March 2015. 